ARTICLE

Received 23 Jun 2015 | Accepted 6 Oct 2015 | Published 27 Nov 2015

DOI: $10.1038 /$ ncomms 9849

OPEN

\title{
Boron nitride colloidal solutions, ultralight aerogels and freestanding membranes through one-step exfoliation and functionalization
}

Weiwei Lei ${ }^{1}$, Vadym N. Mochalinn ${ }^{2,3}$, Dan Liu', Si Qin ${ }^{1}$, Yury Gogotsi ${ }^{2}$ \& Ying Chen ${ }^{1}$

Manufacturing of aerogels and membranes from hexagonal boron nitride (h-BN) is much more difficult than from graphene or graphene oxides because of the poor dispersibility of h-BN in water, which limits its exfoliation and preparation of colloidal solutions. Here, a simple, one-step mechano-chemical process to exfoliate and functionalize h-BN into highly water-dispersible, few-layer $\mathrm{h}$-BN containing amino groups is presented. The colloidal solutions of few-layer h-BN can have unprecedentedly high concentrations, up to $30 \mathrm{mg} \mathrm{ml}^{-1}$, and are stable for up to several months. They can be used to produce ultralight aerogels with a density of $1.4 \mathrm{mg} \mathrm{cm}^{-3}$, which is $\sim 1,500$ times less than bulk $\mathrm{h}-\mathrm{BN}$, and freestanding membranes simply by cryodrying and filtration, respectively. The material shows strong blue light emission under ultraviolet excitation, in both dispersed and dry state.

\footnotetext{
${ }^{1}$ Institute for Frontier Materials, Deakin University, 75 Pigdons Road, Waurn Ponds, Geelong, Victoria 3216, Australia. ${ }^{2}$ A. J. Drexel Nanomaterials Institute, and Materials Science and Engineering Department, Drexel University, 3141 Chestnut Street, Philadelphia, Pennsylvania 19104, USA. ${ }^{3}$ Department of Chemistry, Missouri University of Science \& Technology, 1870 Miner Circle, Rolla, Missouri 65409, USA. Correspondence and requests for materials should be addressed to W.L. (email: weiwei.lei@deakin.edu.au) or to Y.C. (email: ian.chen@deakin.edu.au).
} 
T wo-dimensional boron nitride (BN) nanosheets, also called 'white graphene' or 'non-carbon graphene', consist of a few layers of alternating boron and nitrogen atoms in a hexagonal arrangement. Their mechanical, thermal and electronic properties are attractive for applications in polymer matrix composites $^{1,2}$, hydrogen storage ${ }^{3}$, field emitters ${ }^{4}$, electrocatalysts ${ }^{5}$ and sorbents ${ }^{6,7}$. In addition, it has been demonstrated that BN single crystals, unlike carbon materials, have strong luminescence in the ultraviolet range, which makes $\mathrm{BN}$ attractive for blue light and ultraviolet emission ${ }^{8}$.

In recent times, significant efforts have been focused on the isolation and functionalization of $\mathrm{BN}$ nanosheets to achieve better dispersion, which would enable applications in optical devices, biological systems and composites ${ }^{9-11}$. Organic solvents such as $\mathrm{N}, \mathrm{N}$-dimethylformamide have been employed for exfoliation and stabilization of disperse BN nanosheets, through polar-polar interactions between the functional groups and the hexagonal BN (h-BN) surface, using a sonication-centrifugation technique ${ }^{12}$. Lin et al..$^{13}$ have reported that ball-milled h-BN with an increased number of defects can be functionalized with a long alkyl chain amine via Lewis acid-base interactions to produce water-soluble sheet-like particles. They also reported that water dispersion of h-BN nanosheets can be achieved directly by using water as the exfoliating molecule through edge functionalization ${ }^{14}$. In recent times, Sainsbury et al. ${ }^{15}$ obtained hydroxyl-terminated BN nanosheets by the oxidation of exfoliated h-BN nanosheets using a two-step functionalization procedure. However, the concentration of the $\mathrm{h}-\mathrm{BN}$ dispersions was typically below $2 \mathrm{mg} \mathrm{ml}^{-1}$, even after long periods of intense ultrasonication. The low concentration may present an especially severe limitation for the aqueous suspensions preferred in many applications. Therefore, the development of a practical high-yield process to achieve highly water-soluble BN nanomaterials remains a challenge.

Graphene oxide and graphene, in the form of aerogels and membranes ${ }^{16-18}$, have been used as efficient adsorbents for the separation of organic pollutants and oils from water ${ }^{19,20}$, for gas separation $^{21}$ and for molecular and ion selective devices ${ }^{22,23}$. There is potential for h-BN, which is more oxidation and intercalation resistant than $s p^{2}$ carbon, to be used to produce similar structures ${ }^{24-26}$. The h-BN-based materials represent a great advantage for high-temperature applications and extreme environments. However, it is very difficult to achieve aqueous dispersion of h-BN using conventional routes. In recent times, BN foams assembled from nanosheets showed excellent thermal stability, super-elasticity and very low electrical permittivity, but the assembly process required the use of templates, high temperature and vacuum, hazardous and toxic chemicals, and sophisticated fabrication techniques ${ }^{27,28}$.

Here we present a simple and efficient one-step method for the preparation and functionalization of few-layer $\mathrm{BN}$ by solid-state ball milling of commercially available $\mathrm{h}-\mathrm{BN}$ and urea powder. This ambient temperature method has several advantages, including scalability for mass production, low cost, high yield and does not require the use of organic solvents, catalysts, substrates or vacuum systems. The as-produced few-layer BN shows unprecedentedly high dispersibility in water, yielding stable colloidal solutions with concentrations up to $30 \mathrm{mg} \mathrm{ml}^{-1}$. A urea-assisted exfoliation and functionalization mechanism is proposed to explain the formation of the water-soluble few-layer $\mathrm{BN}$. Ultralight $\mathrm{BN}$ aerogels can be produced by cryodrying and thin freestanding membranes fabricated by filtration of the twodimensional few-layer BN dispersions. Both colloidal solutions and freestanding membranes show strong ultraviolet and blue light luminescence.

\section{Results}

Synthesis and characterization. We have developed a one-step method for the preparation and functionalization of few-layer BN based on urea-assisted solid exfoliation of commercially available h-BN (Fig. 1a). After ball milling and washing with water to remove urea, few-layer $\mathrm{BN}$ can be readily dispersed in water without sonication, to form stable colloidal solutions at different concentrations (Fig. 1b and Supplementary Fig. 1). The concentration of the suspensions can reach up to $30 \mathrm{mg} \mathrm{ml}^{-1}$ (Fig. 1b), the highest ever value reported for $\mathrm{BN}$ water suspensions ${ }^{10-15}$. The suspensions with a high concentration up to $30 \mathrm{mg} \mathrm{ml}^{-1}$ exhibited a milky and light yellow appearance compared with the low-concentration suspensions, possibly due to a relatively high concentration of amino group ${ }^{29,30}$. The yield of the exfoliated BN sheets in the proposed urea-assisted, highenergy ball mill processing was as high as $85 \%$. The path of a red laser beam can be clearly seen through all the dispersions due to light scattering by the few-layer BN colloid (Tyndall effect), as shown in Fig. 1b. The aqueous BN dispersion with the highest
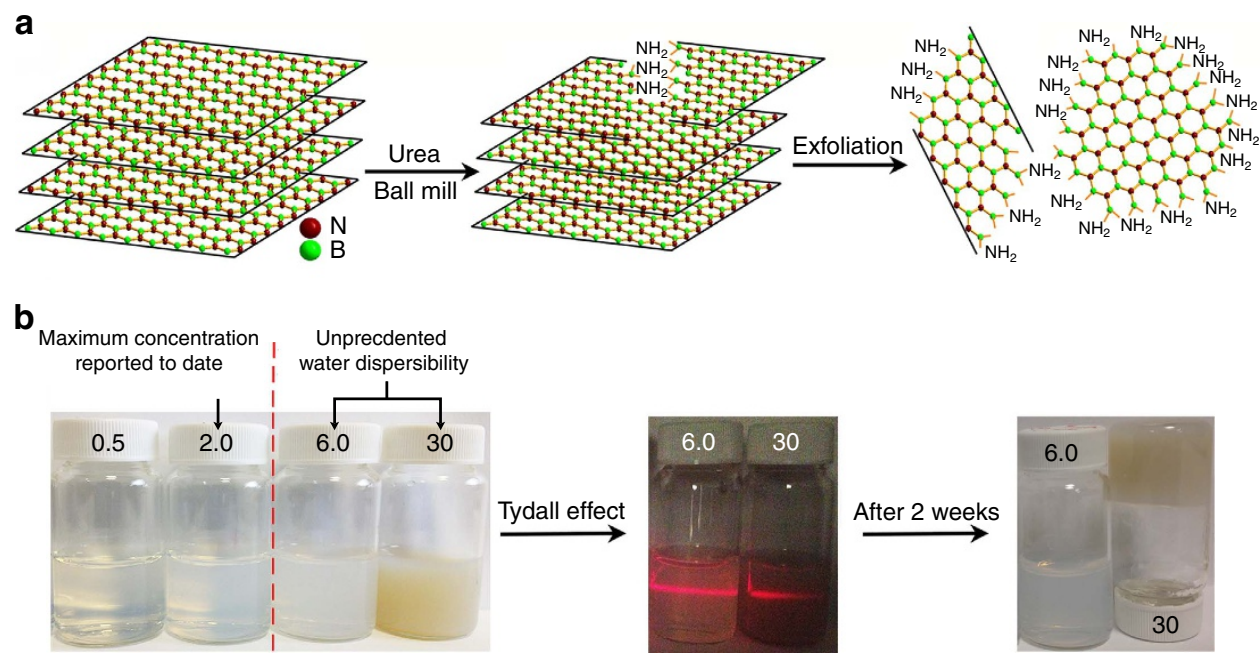

Figure 1 | Schematic illustration of the exfoliation and dispersion process. (a) Schematic illustration of the exfoliation. (b) Photos of as-prepared colloidal solutions of few-layer BN with concentrations of $0.5,2.0,6.0$ and $30 \mathrm{mg} \mathrm{ml}^{-1}$, respectively, and demonstration of the Tyndall effect and stability of the colloidal solutions. 
concentration $\left(30 \mathrm{mg} \mathrm{ml}^{-1}\right)$ transformed into a hydrogel within 2 weeks when left undisturbed (Fig. 1b). Based on the Doi and Edwards theory ${ }^{31}$, the sol-gel transformation can be attributed to the action of van der Waals interparticle forces at the contact points, which will lead to formation of a three-dimensional (3D) continuous network in highly concentrated nanosheet dispersions. Scanning electron microscopy (SEM) images show that the pristine non-milled h-BN has particles with lateral dimensions of $2-10 \mu \mathrm{m}$, with smooth surfaces and edges, which are stacked together (Supplementary Fig. 2a). In contrast, the dispersed particles have a fluffy and lamellar morphology. The images of these samples, formed by evaporating a drop of diluted aqueous suspension on a $\mathrm{Si}$ wafer can be seen in Supplementary Fig. 2b,c. The size and the thickness of the dispersed few-layer BN sheets are reduced compared with the pristine sheets.

Figure 2a shows the transmission electron microscopy (TEM) image of the few-layered $\mathrm{BN}$ prepared by evaporating a drop of diluted colloidal solution on a carbon-coated copper grid. The TEM image suggests that the $\mathrm{BN}$ particles are flat and quite thin. The selected area electron diffraction pattern shows two characteristic diffraction rings $((002)$ and (100)) of layered BN (Fig. 2a inset). The selected area electron diffraction image has a slightly pronounced hexagonal pattern, due to the few-layer stacking of h-BN. The high-resolution TEM (HRTEM; Fig. 2b,c) images at the edges of few-layered BN clearly show three and six parallel fringes corresponding to three and six stacked layers in the samples. As seen in the HRTEM and the fast Fourier transform images in Fig. 2d, the structure of the few-layer BN remains ordered after ball milling. The thickness and lateral size distributions were statistically evaluated by HRTEM measurements on 100 sheets (Supplementary Fig. 3). Eighty-three per cent of the BN sheets were $<2.5 \mathrm{~nm}$ thick and around $100 \mathrm{~nm}$ in lateral size. The nanometre-scale thickness of few-layered $\mathrm{BN}$ was further confirmed by atomic force microscopy (Fig. 2e). Figure $2 f$ suggests that the thickness of the few-layer $\mathrm{BN}$ is about $2 \mathrm{~nm}$ (five to six monolayers). The structure of few-layer $\mathrm{BN}$ was further investigated by X-ray diffraction (XRD) analysis. As shown in Fig. 2g, two main characteristic diffraction peaks can be observed at $26.2^{\circ}$ and $42.8^{\circ}$, arising from (002) and (100) planes of few-layer BN, respectively. Compared with the pristine h-BN, the (002) and (100) peaks of few-layer BN show a remarkably reduced intensity and dramatically broadened width, indicating the presence of thin $\mathrm{BN}$ sheets and much less extended/ordered stacking in the $c$ direction.

The Fourier transform infrared (FTIR) spectrum shows an additional peak at $3,240 \mathrm{~cm}^{-1}$ due to the $\mathrm{N}-\mathrm{H}$ stretching vibration $^{10,32}$, whereas pristine $\mathrm{h}-\mathrm{BN}$ exhibits only the characteristic peaks of in-plane $\mathrm{B}-\mathrm{N}$ stretching vibrations at $\sim 1,364 \mathrm{~cm}^{-1}$ and out-of-plane $\mathrm{B}-\mathrm{N}-\mathrm{B}$ bending vibrations at $\sim 1,746 \mathrm{~cm}^{-1}$ (Supplementary Fig. 4$)^{33}$. The shoulder at $3,411 \mathrm{~cm}^{-1}$ can be ascribed to $\mathrm{O}-\mathrm{H}$ stretching vibrations due to the washing in water after ball milling ${ }^{10,14,15}$. The appearance of $\mathrm{N}-\mathrm{H}$ stretching vibrations indicates that functionalization has occurred. Functional groups such as $\mathrm{NH}_{2}$ from urea have been created and bonded to the defect sites and edges of few-layer BN. In addition, the strong pungent smell and the readings of an ammonia sensor on opening the milling jar further demonstrate that ammonia gas and/or low-molecular-weight amines were formed as intermediates in the mechano-chemical process. Although the recently reported hydroxyl functionalized BN nanosheets prepared via $\mathrm{NaOH}$-assisted ball milling had very low dispersibility in water with highest achievable concentrations $<1 \mathrm{mg} \mathrm{ml}^{-1}$ (ref. 11), the functionalized few-layer BN reported here forms aqueous colloidal solutions with concentration up to $30 \mathrm{mg} \mathrm{ml}^{-1}$.

Dispersions of the pristine $\mathrm{BN}$ and few-layered $\mathrm{BN}$ were investigated using zeta potential measurements ${ }^{34}$. Both of them exhibit highly negative potential values, $-44 \pm 3$ and $-34 \pm 4 \mathrm{mV}$, respectively, consistent with the reported values for $\mathrm{BN}$ materials due to the $\mathrm{B}-\mathrm{O}-\mathrm{H}$ and $\mathrm{N}-\mathrm{O}-\mathrm{H}$ generated on h-BN in water ${ }^{35,36}$. It has been reported that the hydrophilicity, shape, size distribution and zeta potential of particles are all important factors governing their dispersibility in water and stability of formed dispersion ${ }^{35,36}$. The excellent hydrophilicity and narrow size distribution are essential to the good dispersibility, whereas high zeta potential helps to stabilize the dispersion. Although zeta potential of pristine $\mathrm{BN}$ is $-44 \pm 3 \mathrm{mV}$, it cannot disperse in water well due to its

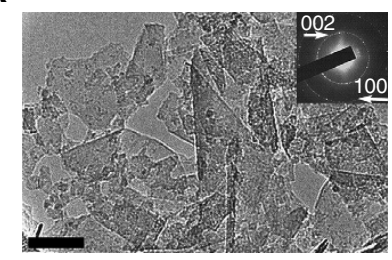

e

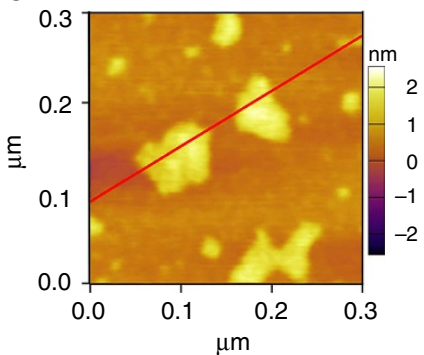

b

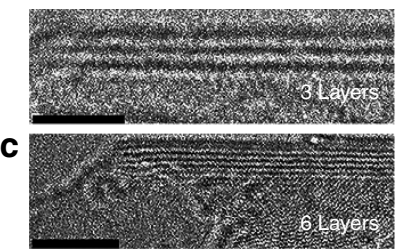

f

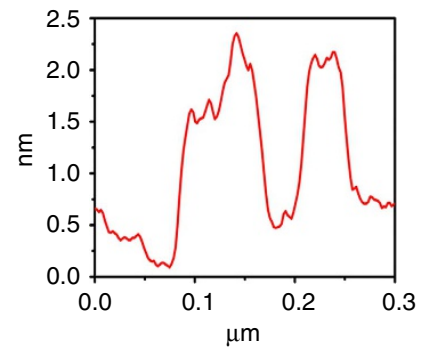

d

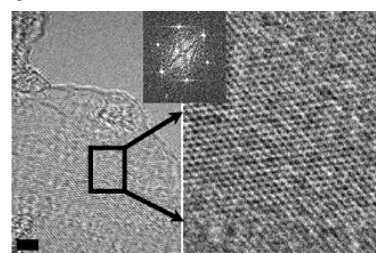

g

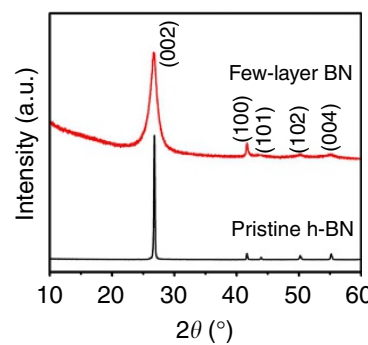

Figure 2 | Exfoliated few-layer BN. (a) TEM image of few-layer BN, with the selected-area electron diffraction pattern (inset) indicating a layered BN structure. Scale bar, $50 \mathrm{~nm}$. (b,c) HRTEM images of the edge folding of two few-layer BN sheets with three and six BN layers, respectively. Scale bars, $2 \mathrm{~nm}$ (b) and $5 \mathrm{~nm}$ (c). (d) HRTEM and the fast Fourier transform images of a few-layer BN sheet. Scale bar, $2 \mathrm{~nm}$. (e,f) Atomic force microscopy image and corresponding line-scan profile of few-layer BN. (g) XRD patterns of few-layer BN and pristine h-BN. 
hydrophobicity and large particle size (Supplementary Figs 5a and $2 \mathrm{a})^{35}$. The few-layer $\mathrm{BN}$ exhibits a somewhat decreased absolute value of zeta potential $(-34 \pm 4 \mathrm{mV})$ after functionalization with $\mathrm{NH}_{2}$ groups. The reduced negative zeta potential after functionalization with $\mathrm{NH}_{2}$ groups is due to partial neutralization effect between positively charged $\mathrm{NH}_{3}^{+}$groups and negatively charged oxygen-containing groups formed on $\mathrm{BN}$ surface on contact with water. Thus, although the absolute value of zeta potential decreased compared with pristine $\mathrm{BN}$, it is still quite high and the few-layer $\mathrm{BN}$ demonstrates excellent water dispersibility compared with pristine $\mathrm{BN}$. In addition to electrostatic stabilization, this result is attributed to the improved hydrophilicity, uniform thickness and a narrower size distribution after ball milling and the attachment of $\mathrm{NH}_{2}$ groups (Fig. 2 and Supplementary Fig. 5b).

The high-resolution $\mathrm{B} 1 \mathrm{~s}$ and $\mathrm{N}$ 1s X-ray photoelectron spectroscopy (XPS) results are shown in Supplementary Fig. 6. In Supplementary Fig. 6a, the component at $190.4 \mathrm{eV}$ corresponds to $\mathrm{B}-\mathrm{N}$ bonds in $\mathrm{h}-\mathrm{BN}$ and the shoulder $(191.5 \mathrm{eV})$ should be attributed to the $\mathrm{B}$ atoms in $\mathrm{B}-\mathrm{O}$ bonds formed due to the exposure of the few-layered $\mathrm{BN}$ to ambient environment and the hydroxyl groups attached to defects along the edges of h-BN during washing in water after ball milling ${ }^{94,37}$. In addition, the $\mathrm{B}-\mathrm{O}$ bonds may originate from the pristine $\mathrm{h}-\mathrm{BN}$. In the synthesis process of $\mathrm{h}-\mathrm{BN}$, oxygen-containing boron trioxide $\left(\mathrm{B}_{2} \mathrm{O}_{3}\right)$ or boric acid are typically used as a precursor. It is possible that the h-BN particles used in the present study contained small amounts of oxygen. In Supplementary Fig. $6 \mathrm{~b}$, the main peak with a binding energy of $398.2 \mathrm{eV}$ in the $\mathrm{N} 1 \mathrm{~s}$ spectrum corresponds to $\mathrm{N}-\mathrm{B}$ bonds in h-BN; a shoulder can be deconvoluted at $399.6 \mathrm{eV}$ corresponding to $\mathrm{N}-\mathrm{H}$ bonds, which is another evidence of the presence of amino group ${ }^{37}$. The amount of the attached $\mathrm{NH}_{2}$ groups was evaluated from the thermogravimetric analysis (TGA), which suggests $\sim 1.3 \mathrm{wt} \%$ content of $\mathrm{NH}_{2}$ groups in the functionalized h-BN sheets (Supplementary Fig. 7). The presence of urea-induced $\mathrm{N}-\mathrm{H}$-containing groups render the few-layer $\mathrm{BN}$ hydrophilic, with an average observed contact angle of $43 \pm 1^{\circ}$ resulting in a high dispersibility in water, whereas the pristine $\mathrm{BN}$ has an average contact angle of $115 \pm 4^{\circ}$ (Supplementary Fig. 5). Thus, $\mathrm{NH}_{2}$ species produced from urea and attached to $\mathrm{BN}$ during ball milling are strongly beneficial to the colloidal properties of the few-layer $\mathrm{BN}$, providing stable aqueous dispersions. To confirm this, the pristine h-BN was milled with $\mathrm{NaCl}$, which has previously been used to de-agglomerate nanodiamond ${ }^{38}$, under the same milling conditions. Compared with the sample milled with urea, there was no apparent water solubility of h-BN after milling with $\mathrm{NaCl}$ (Supplementary Fig. 8). This shows the importance of the presence of urea in the mechano-chemical process of exfoliation and functionalization of $\mathrm{BN}$, yielding hydrophilic few-layer $\mathrm{BN}$ readily dispersible in water.

Theoretical simulations. Interactions of amino groups with an h-BN monolayer were studied by density functional theory (DFT) calculations. The following sites for the adsorption of $\mathrm{NH}_{2}$ on the h-BN single layer were considered: (1) $\mathrm{N}$ atoms at the zigzag edge of BN (Fig. 3a), (2) B atoms at the edge of BN (Fig. 3b), (3) basalplane $\mathrm{N}$ atoms (Fig. 3c) and (4) basal-plane B atoms (Fig. 3d). The corresponding bond lengths and angles, as well as final enthalpy values for the most stable structures of each configuration produced by geometry optimization, are listed in Supplementary Table 1.

As expected, due to the dangling bond saturation, the structures where an $\mathrm{NH}_{2}$ group is attached to the zigzag edge of h-BN (Fig. 3a,b) are more stable than the structures where the $\mathrm{NH}_{2}$ is attached to the atoms in the basal plane (Fig. 3e). For $\mathrm{NH}_{2}$ attached to an edge $\mathrm{N}$ atom (Fig. 3a), there is evidence of additional interactions between $\mathrm{H}$ atoms of the amino group and the nearby $\mathrm{N}$ atoms, potentially hydrogen bonding, indicated by dashed lines in Fig. 3a. This is supported by an unusually short $\mathrm{N}-\mathrm{N}$ distance between the $\mathrm{N}$ atom of the $\mathrm{NH}_{2}$ and the edge $\mathrm{N}$ atom of h-BN, as well as a large $\mathrm{H}-\mathrm{N}-\mathrm{H}$ valence angle of $\mathrm{NH}_{2}$ (Supplementary Table 1). These interactions additionally stabilize the structure. Still, bonding of $\mathrm{NH}_{2}$ to $\mathrm{B}$ terminating the edge resulted in the most stable and preferred configuration of the four considered, as shown by the enthalpy of reaction (Fig. 3e).

For both structures with basal attachment of $\mathrm{NH}_{2}$ (Fig. 3c,d), by contrast, the formed bonds are longer (Supplementary Table 1), an indication of steric hindrance to $\mathrm{NH}_{2}$ bonding. The basal $\mathrm{B}$ atom bonded to $\mathrm{NH}_{2}$ comes slightly off the $\mathrm{BN}$ plane, signifying its transition from planar to tetrahedral bonding. The configuration with $\mathrm{NH}_{2}$ attached to a basal $\mathrm{N}$ atom of h-BN is the least energetically stable (Supplementary Table 1) and its formation is unfavourable, as indicated by a positive $\Delta H_{\mathrm{r}}$ value (Fig. 3e). The $\mathrm{H}-\mathrm{N}-\mathrm{H}$ angle of $\mathrm{NH}_{2}$ in this configuration (Fig. 3c) is close to unbonded $\mathrm{NH}_{2}$ (Supplementary Table 1) and the $\mathrm{HNH}$ plane is oriented more in parallel to the h-BN plane in contrast to its nearly perpendicular orientation in another basal configuration (Fig. 3d). These are indicators that $\mathrm{NH}_{2}$ adsorption, not covalent bonding, may be the most probable mechanism in this case. In fact, we observed that in several simulations when the
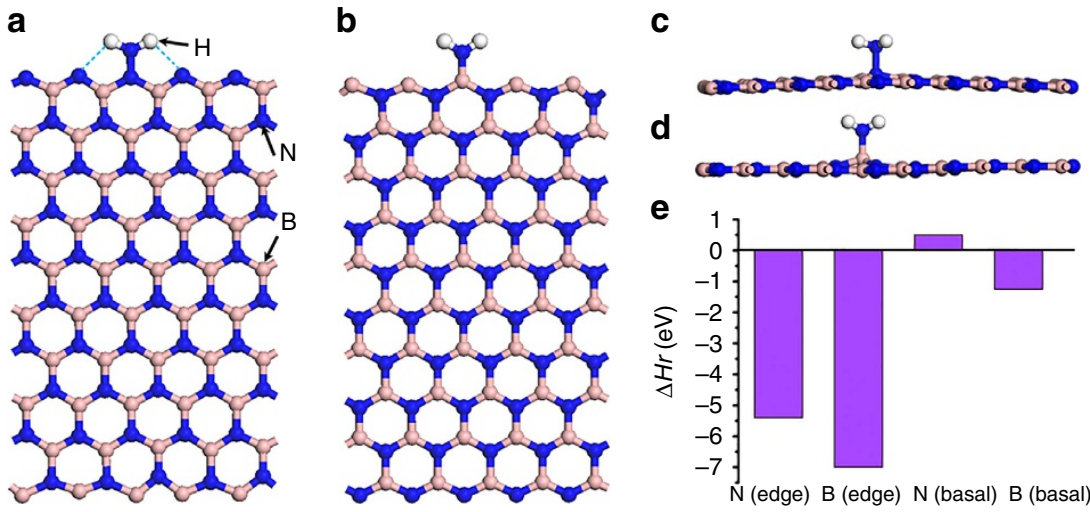

Figure 3 | Optimized configurations of $\mathbf{B N}$-amine interaction. (a) $\mathrm{An} \mathrm{NH}_{2}$ at the nitrogen edge atom, (b) an $\mathrm{NH}_{2}$ at the boron zigzag edge atom, (c) an $\mathrm{NH}_{2}$ on a basal-plane $\mathrm{N}$ atom and (d) an $\mathrm{NH}_{2}$ on a basal-plane $\mathrm{B}$ atom. (e) Reaction enthalpies for the different optimized geometries of the configurations (a-d). 
$\mathrm{NH}_{2}$ group was not precisely positioned above the basal $\mathrm{N}$ atom, it moved and formed covalent bond with a neighbouring $\mathrm{B}$ atom, ending up in a configuration similar to Fig. 3d. Thus, DFT modelling confirms that $\mathrm{NH}_{2}$ groups can be adsorbed and chemically bonded to h-BN. Most preferred sites for chemical bonding are the edge $\mathrm{B}$ and $\mathrm{N}$ atoms, with covalent bonding to the edge $\mathrm{B}$ atom being the most favourable. On the basal BN plane $\mathrm{NH}_{2}$ bonding to a $\mathrm{B}$ atom is possible, whereas the reaction of $\mathrm{NH}_{2}$ with a basal $\mathrm{N}$ atom is not energetically favourable.

Mechanism of exfoliation and functionalization of h-BN. Based on the above structural characteristics and analysis, as well as DFT calculations, we propose the following mechanism for the one-step exfoliation and functionalization of few-layer BN. The exfoliation process involves two stages.

At the beginning of ball milling, the sizes of pristine h-BN and urea particles are reduced due to the high shear forces and highenergy collisions with the stainless steel balls. In this process, BN and urea particles are thoroughly mixed together and some small urea particles adsorb on the BN surface. The layered BN structure is wedged by the small urea particles, resulting in a slight increase of the interlayer spacing (Supplementary Fig. 9). Some urea molecules may intercalate into the BN structure from the edges of $\mathrm{BN}$ layers during high-energy ball impacts. Size reduction and exfoliation were confirmed by the XRD patterns of the products after $20 \mathrm{~h}$ milling (Supplementary Fig. 9) without washing with water, which show dramatically broadened and less intense (002) diffraction. An excess of urea over $\mathrm{BN}$ ensured sufficient intercalation of urea molecules into the BN structure ${ }^{39-41}$.

Mechano-chemical processes powered by the energy of colliding balls lead to decomposition of urea and chemical bonding of $\mathrm{NH}_{2}$ groups to few-layer $\mathrm{BN}^{42}$. This results in the attachment of $\mathrm{NH}_{2}$ to $\mathrm{BN}$ edges and defects, as confirmed by FTIR and XPS spectra and TGA (Supplementary Figs 4, 6 and 7), and prevents restacking of $\mathrm{BN}$ sheets. The DFT calculations demonstrate that the $\mathrm{NH}_{2}$ groups can be adsorbed on the $\mathrm{B}$ sites of the $\mathrm{BN}$ surface and easily form bonds with the edge $\mathrm{B}$ and $\mathrm{N}$ sites, rendering few-layer $\mathrm{BN}$ hydrophilic and assisting in its stabilization in aqueous dispersions.

BN aerogels and membranes. Functional aerogels and transparent thin membranes ${ }^{16-18}$ have been extensively developed, to exploit the properties of nanomaterials over a wide range of applications. Ultralight $\mathrm{BN}$ aerogels and freestanding membranes can be produced via cryodrying and filtration of few-layer BN suspensions, respectively. A BN aerogel can be easily prepared by a one-step cryo-desiccation of the BN aqueous dispersion (Fig. 4). The ultralow density of the aerogel was demonstrated by placing it on the delicate spike of a plant (Fig. 4a), as well as adhering to a nearly vertical beaker wall by electrostatic attraction (Fig. 4b). The density of the aerogel was estimated, including the density of the air occupying the pores, to be in the range from 1.4 to $20 \mathrm{mg} \mathrm{cm}^{-3}$, depending on the concentration of few-layer BN in the aqueous dispersion. The $1.4 \mathrm{mg} \mathrm{cm}^{-3} \mathrm{BN}$ aerogel is the lightest reported among BN-based materials and much lighter than many carbon and other aerogels ${ }^{43-47}$. In addition, the Brunauer-Emmett-Teller analysis of low-temperature nitrogen adsorption isotherms shows that the $\mathrm{BN}$ aerogel has a high specific surface area of $273 \mathrm{~m}^{2} \mathrm{~g}^{-1}$ (Supplementary Fig. 10). Barret-Johner-Halenda calculations give a broad pore size distribution in the range of $2-50 \mathrm{~nm}$. Larger pores were probably present, but they could not be measured by gas adsorption. The heavier sample, with a density of $20 \mathrm{mg} \mathrm{cm}^{-3}$, distorted the seed spike more (Fig. 4c). The corresponding SEM investigations revealed that the $\mathrm{BN}$ low-density aerogel has a loose $3 \mathrm{D}$ structure of pores connected by thin walls, whereas the higher density aerogel has larger 'sheet-like' structure of thicker connecting walls (Fig. 4d,e). The ultralow weight together with low electrical conductivity and high thermal and chemical stability of $\mathrm{BN}$ enable potential applications of these gels in insulation, dielectrics and ultralight materials ${ }^{24}$.

BN membranes were directly fabricated using vacuum-assisted filtration of the few-layer $\mathrm{BN}$ dispersion. The membrane can be readily peeled off from the filter and retains its mechanical a
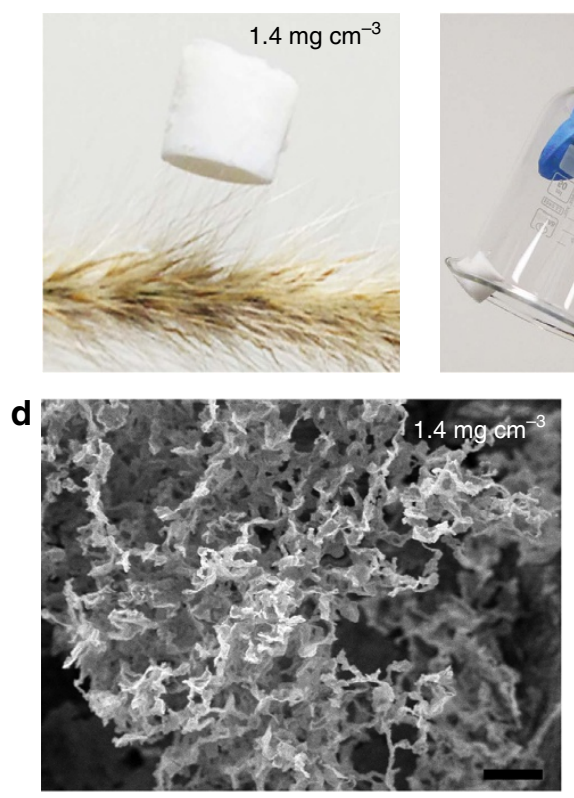

b

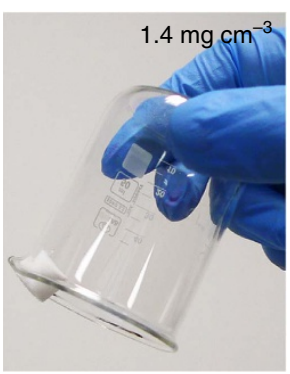

C
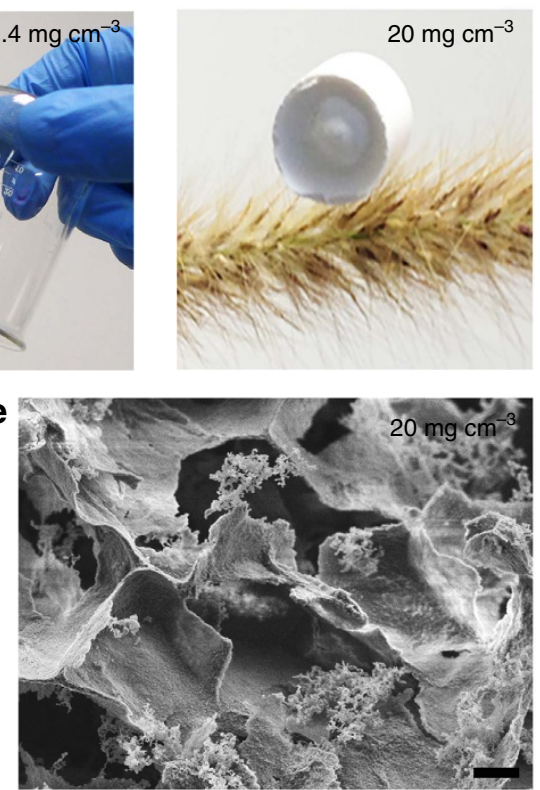

Figure 4 | BN aerogel. (a) Photo of an aerogel with a low density $\left(1.4 \mathrm{mg} \mathrm{cm}^{-3}\right.$ ) placed on the spike of a plant. (b) Photo of an aerogel adhering to a beaker wall. (c) Photo of an aerogel of a higher density $\left(20 \mathrm{mg} \mathrm{cm}^{-3}\right)$ pressing the spike of a plant. (d,e) SEM images of aerogels with densities of 1.4 and $20 \mathrm{mg} \mathrm{cm}^{-3}$, respectively. Scale bars, $2 \mu \mathrm{m}(\mathbf{d}, \mathbf{e})$. 
a

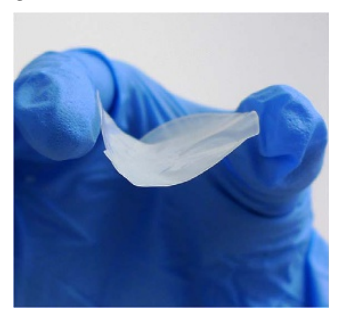

b

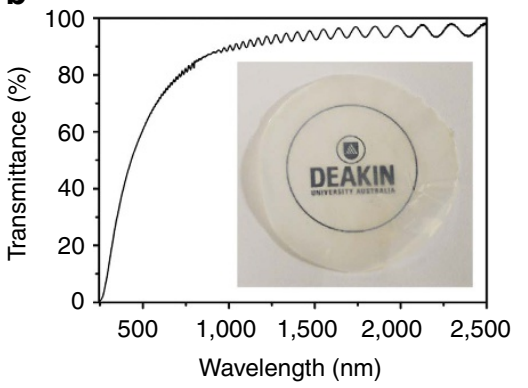

C

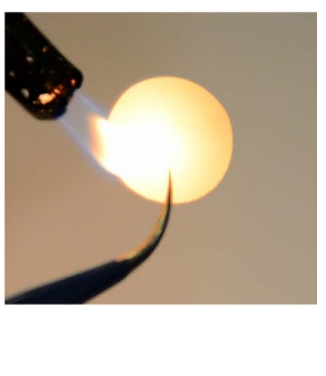

d

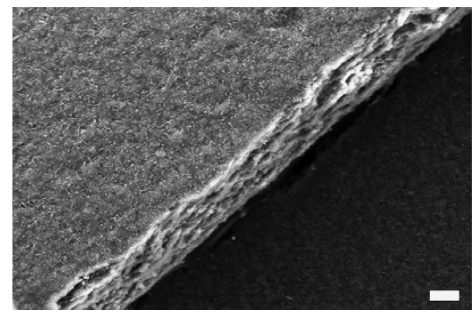

e

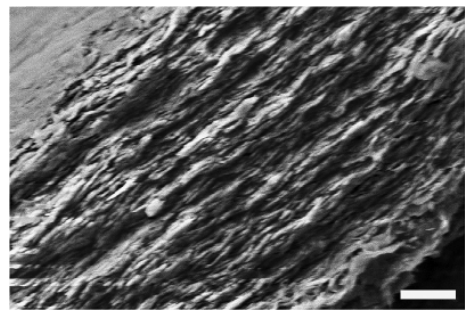

Figure 5 | BN membranes. (a) Photo of the freestanding BN membrane. (b) Optical transmittance of the freestanding BN membrane and inset is a photo of the BN membrane placed over a paper with a printed logo. (c) Photo of a BN membrane held in the flame in ambient air. SEM images of the basal (d) and fracture surface (e) of the BN membrane. Scale bars, $2 \mu \mathrm{m}$ (d) and $1 \mu \mathrm{m}(\mathbf{e})$.

integrity, while being flexible in the freestanding state (Fig. 5a). This ultrathin membrane is nearly transparent to visible light as shown in Fig. 5b. The optical transparency of the membrane can be attributed to its thickness and the inherently low extinction coefficient of $\mathrm{BN}$ in the visible range ${ }^{48}$-in striking contrast to graphene. Furthermore, the thickness of the membrane can be tuned in the range of $0.2-100 \mu \mathrm{m}$ by filtering the desired quantity of few-layer BN dispersion with a fixed concentration. The optical transmittance of a freestanding membrane $(\sim 10 \mu \mathrm{m}$ thick according to SEM) was measured by ultraviolet-visible-near infrared spectrometer and is shown in Fig. 5b. The membrane completely absorbs ultraviolet light, while retaining high optical transmittance in the visible range above $500 \mathrm{~nm}$ and up to $95 \%$ transmittance in the near-infrared range. The oscillations in the transmittance in the near-infrared region are a result of the interference of light reflected at the air-membrane and membrane-substrate interfaces. The pronounced and clean interference pattern indicates a relatively smooth surface and uniform thickness of the membrane ${ }^{49}$. These optical properties indicate potential applications of $\mathrm{BN}$ membranes for ultraviolet shielding/protection. Moreover, the $\mathrm{BN}$ membrane is also fireresistant and does not burn in the flame of a gas lighter in ambient air (Fig. 5c). BN membranes are much more thermally stable and have a higher resistance to oxidation than any carbonbased material. Figure 5d,e show the top surface and crosssectional SEM images of the $\mathrm{BN}$ membrane. The cross-sectional image, as shown in Fig. 5e, reveals a compact stacking of fewlayer BN with uniform thickness $(\sim 10 \mu \mathrm{m})$.

Photoluminescence spectra. Figure 6 shows the photoluminescence $(\mathrm{PL})$ spectra of a few-layer $\mathrm{BN}$ dispersion in water and of a freestanding membrane recorded with an excitation wavelength of $200 \mathrm{~nm}$. As shown in Fig. 6, a strong ultraviolet PL emission peak at $275 \mathrm{~nm}$ can be seen in both samples, attributed to deep levels in the band gap from intrinsic $\mathrm{B}$ or $\mathrm{N}$ defects, which is similar to previously reported results for $\mathrm{BN}$ nanosheets, bamboo-like multiwall nanotubes and nanohorns containing bent $\mathrm{BN}$ layers ${ }^{50-52}$. There is a weak peak at $\sim 385 \mathrm{~nm}$, appearing as a

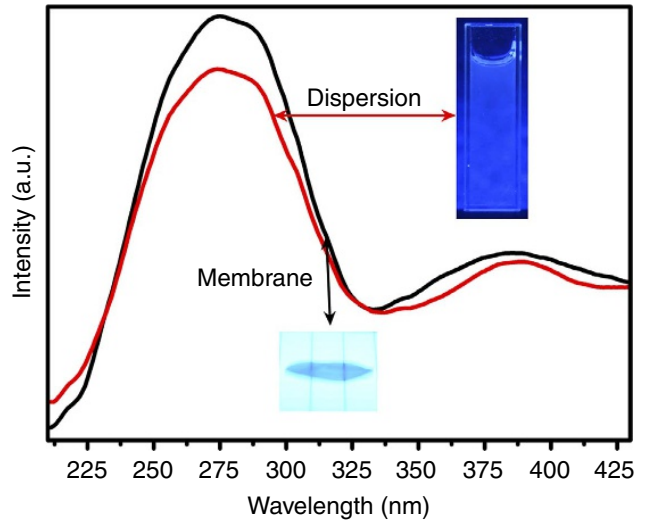

Figure 6 | Photoluminescence of BN. Emission spectra of colloidal solution and a BN membrane under excitation at $200 \mathrm{~nm}$. The insets are the corresponding photos.

shoulder to the main PL peak. This peak may be assigned to impurities (possibly oxygen) or to generic structural defects ${ }^{53-56}$. The digital photographs (Fig. 6 insets) of the aqueous dispersions and freestanding membranes under $365 \mathrm{~nm}$ ultraviolet light further confirm the blue light emission. The strong ultraviolet and blue light emission indicates that few-layer BN dispersions and freestanding membranes may have potential applications in bioimaging, ultraviolet laser emitters and optoelectronic devices.

\section{Discussion}

A simple and high-yield process has been developed to fabricate few-layer $\mathrm{h}-\mathrm{BN}$ with a thickness around $2.5 \mathrm{~nm}$ and lateral dimensions mostly below $100 \mathrm{~nm}$. The procedure involves ureaassisted mechanical exfoliation during which mechano-chemical reactions take place at atmospheric pressure and room temperature. The residual urea can be washed away, resulting in a high concentration of well-dispersed few-layer $\mathrm{BN}$ in water without 
any posttreatment. Ultralight and freestanding aerogels can be produced by cryodrying of the few-layer dispersions, whereas filtration gives thin, high-quality membranes of controllable thickness. Importantly, the as-obtained few-layer BN dispersions and membranes show strong light emission when excited with an ultraviolet source. The stable colloidal aqueous dispersions of few-layer BN, ultralight aerogels and freestanding membranes produced in this study may be highly beneficial for a wide range of applications.

\section{Methods}

Synthesis. In a typical synthesis, h-BN (Momentive Performance Materials Inc.) and urea (Sigma-Aldrich) were mixed together at the weight ratio 1:60 inside a steel milling container using a planetary ball mill (Pulverisette 7, Fritsch) at a rotation speed of 700 r.p.m. for $20 \mathrm{~h}$ at room temperature under nitrogen atmosphere. The high rotation speed provides high power and effective exfoliation of h-BN on a large scale. The urea not only assists the exfoliation but also protects the $\mathrm{BN}$ from excessive mechanical damage, preventing an extensive formation of lattice defects. The variations in weight ratio (1:20 and 1:100) and milling time (10 and $30 \mathrm{~h}$ ) were also investigated. The size and thickness of the few-layer BN particles were reduced at lower h-BN:urea weight ratio or longer milling time. The results are shown in Supplementary Table S2. In this study, the weight ratio of h-BN:urea and milling time were fixed at 1:60 and $20 \mathrm{~h}$, correspondingly, to get $\mathrm{BN}$ sheets with suitable size and thickness for the following preparation of aerogel and membrane. After ball milling, the obtained powders were dissolved in water. The resulting few-layer BN aqueous dispersion was dialysed for 1 week (membrane cutoff: $3,500 \mathrm{kDa}$ ) in de-ionized water, to remove the urea. Stable aqueous dispersions were then obtained. For milling of pristine h-BN with $\mathrm{NaCl}$, the same milling conditions were used. XRD and FTIR spectra were recorded to confirm the absence of any traces of urea and $\mathrm{NaCl}$ in the few-layer $\mathrm{BN}$. The as-prepared hydrogel was directly dehydrated via a freeze-drying process to maintain the $3 \mathrm{D}$ monolith architecture. The BN membrane can be readily fabricated by vacuum filtration of a stable few-layer $\mathrm{BN}$ in water suspension through an Anodisc membrane filter $(47 \mathrm{~mm}$ diameter, $0.02 \mu \mathrm{m}$ pore size, Whatman), followed by drying in air and peeling the membrane off the filter.

Materials characterization. XRD measurements were performed on a Panalytical X'Pert PRO apparatus with $\mathrm{Cu} \mathrm{K} \alpha$ radiation. SEM analysis was performed on a Zeiss Supra 55 VP SEM instrument. The BN samples were sputtered with carbon before imaging. TEM and HRTEM imaging was performed on a JEOL $2100 \mathrm{~F}$ microscope operated at $200 \mathrm{kV}$. Samples were prepared by evaporating a drop of diluted aqueous suspension on a carbon-coated copper grid. The atomic force microscopy measurements were performed on a Cypher atomic force microscope. The FTIR and optical transmittance spectra were recorded using a Nicolet 7199 FTIR spectrometer and Cary 5000 spectrophotometer, respectively. XPS was performed using an ESCALAB 250 instrument equipped with a non-monochromatic $\mathrm{Mg}-\mathrm{K} \alpha \mathrm{X}$-ray source. The thermal behaviour was analysed by TGA on a TA Instruments Q50 TGA thermal analyser at a heating rate of $10^{\circ} \mathrm{C} \mathrm{min}-1$ from room temperature to $800^{\circ} \mathrm{C}$ under nitrogen gas flow. The zeta potential was measured using a Zetasizer Nano ZS90 apparatus from Malvern. The density of BN aerogel was determined by measuring the weight and volume of the aerogel. Nitrogen adsorption and desorption isotherms were obtained using a Tristar 3000 apparatus at $77 \mathrm{~K}$. Contact angle measurements were made by a contact angle goniometer (CAM101, KSV). Contact angle results are presented as average of five to ten measurements at different locations \pm s.d. The emission spectra were recorded on a Fluoromax-4 spectrometer (Horiba Jobin Yvon Inc.).

Theoretical simulations. The analysis of the different geometries was carried out using a pseudopotential plane-wave method within the DFT framework by Accelrys Materials Studio package. The DFT calculations were performed in the generalized gradient approximation with the Perdew-Burke-Ernzerhof functional implemented in CASTEP module of Accelrys Materials Studio. The BN nanoribbon infinite in one direction (labelled c) was constructed using Materials Studio Visualizer. First, a 5,5 BN periodic single wall nanotube was created and the lattice angles were modified from $\alpha=\beta=90^{\circ}, \gamma=120^{\circ}$ to $\alpha=\beta=\gamma=90^{\circ}$. The structure was then converted into a $1 \times 1 \times 5(a \times b \times c)$ supercell. The nanotube was cut by manually removing a row of $\mathrm{B}-\mathrm{N}$ bonds along the $b$-direction of the cell. The dimensions of the cell were increased to $22.0 \times 22.00 \times 12.75 \AA$ $(a \times b \times c)$, followed by quick geometry optimization in the FORCITE module with Dreiding forcefield, yielding a flat $\mathrm{BN}$ ribbon formed of $9 \times 5$ fused $\mathrm{BN}$ hexagonal rings, infinite in the $c$-direction and with zigzag edges. $\mathrm{N}$ atoms were exposed on one edge and B atoms exposed on the opposite. The cell dimensions were large enough to guarantee no interactions between the system and its periodic images, as the separation between any two atoms in the cell and their adjacent images was always larger than $10 \AA$. Finally, the geometry of an h-BN nanosheet prepared from periodic cells was carefully optimized with CASTEP. Following this, five cells of the same geometry and dimensions $(22.00 \times 22.00 \times 12.75 \AA)$, containing h-BN terminated by $\mathrm{NH}_{2}$ groups on the edge and basal B and $\mathrm{N}$ atoms, as well as a cell with just one $\mathrm{NH}_{2}$ group in the centre, were constructed.

Geometry of all systems was optimized using same settings in CASTEP (no cell optimization, energy cut-off $250.0 \mathrm{eV}, 1 \times 1 \times 2 k$-points on Monkhorst-Pack grid) with convergence tolerance $1.0 \times 10^{-5} \mathrm{eV}$ per atom (energy), $0.03 \mathrm{eV} \AA^{-1}$ (force), $0.05 \mathrm{GPa}$ (stress) and $0.001 \AA$ (displacement). The final enthalpy calculated by CASTEP was used as a measure of stability of the optimized structures. The enthalpy of reaction of an $\mathrm{NH}_{2}$ group with $\mathrm{BN}$ ribbon was calculated as

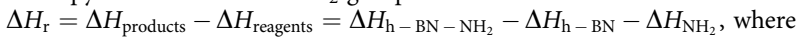
$\Delta H_{\mathrm{h}}-\mathrm{BN}-\mathrm{NH}_{2}$ is the final enthalpy of an h-BN-NH $\mathrm{H}_{2}$ structure, $\Delta H_{\mathrm{h}-\mathrm{BN}}$ and $\Delta H_{\mathrm{NH}_{2}}$ are final enthalpies of h-BN nanoribbon and $\mathrm{NH}_{2}$ group placed in a periodic box of same dimensions and calculated using same settings, respectively.

\section{References}

1. Wang, X., Zhi, C., Weng, Q., Bando, Y. \& Golberg, D. Boron nitride nanosheets: novel syntheses and applications in polymeric composites. J. Phys. Conf. Ser. 471, 012003 (2013).

2. Liu, D. et al. Multifunctional polymer/porous boron nitride nanosheet membranes for superior trapping emulsified oils and organic molecules. Adv. Mater. Interfaces 2, 1500228 (2015)

3. Lei, W. et al. Oxygen-doped boron nitride nanosheets with excellent performance in hydrogen storage. Nano Energy 6, 219-224 (2014).

4. Chen, Z.-G. \& Zou, J. Field emitters: ultrathin BN nanosheets protruded from BN fibers. J. Mater. Chem. 21, 1191-1195 (2011).

5. Uosaki, K. et al. Boron nitride nanosheet on gold as an electrocatalyst for oxygen reduction reaction: Theoretical suggestion and experimental proof. J. Am. Chem. Soc. 136, 6542-6545 (2014).

6. Lei, W., Portehault, D., Liu, D., Qin, S. \& Chen, Y. Porous boron nitride nanosheets for effective water cleaning. Nat. Commun. 4, 1777 (2013).

7. Liu, D., Lei, W., Qin, S. \& Chen, Y. Template-free synthesis of functional 3D BN architecture for removal of dyes from water. Sci. Rep. 4, 4453 (2014)

8. Watanabe, K., Taniguchi, T. \& Kanda, H. Direct-bandgap properties and evidence for ultraviolet lasing of hexagonal boron nitride single crystal. Nat. Mater. 3, 404-409 (2004).

9. Marsh, K. L., Souliman, M. \& Kaner, R. B. Co-solvent exfoliation and suspension of hexagonal boron nitride. Chem. Commun. 51, 187-190 (2015).

10. Weng, Q. et al. Highly water-soluble, porous, and biocompatible boron nitrides for anticancer drug delivery. ACS Nano 8, 6123-6130 (2014).

11. Lee, D. et al. Scalable exfoliation process for highly soluble boron nitride nanoplatelets by hydroxide-assisted ball milling. Nano Lett. 15, 1238-1244 (2015).

12. Zhi, C., Bando, Y., Tang, C., Kuwahara, H. \& Golberg, D. Large-scale fabrication of boron nitride nanosheets and their utilization in polymeric composites with improved thermal and mechanical properties. Adv. Mater. 21, 2889-2893 (2009).

13. Lin, Y., Williams, T. V., Cao, W., Elsayed-Ali, H. E. \& Connell, J. W. Defect functionalization of hexagonal boron nitride nanosheets. J. Phys. Chem. C 114, 17434-17439 (2010).

14. Lin, Y. et al. Aqueous dispersions of few-layered and monolayered hexagonal boron nitride nanosheets from sonication-assisted hydrolysis: critical role of water. J. Phys. Chem. C 115, 2679-2685 (2011).

15. Sainsbury, T. et al. Oxygen radical functionalization of boron nitride nanosheets. J. Am. Chem. Soc. 134, 18758-18771 (2012).

16. Hu, H., Zhao, Z., Gogotsi, Y. \& Qiu, J. Compressible carbon nanotubegraphene hybrid aerogels with superhydrophobicity and superoleophilicity for oil sorption. Environ. Sci. Technol. Lett. 1, 214-220 (2014).

17. Hu, H., Zhao, Z., Wan, W., Gogotsi, Y. \& Qiu, J. Ultralight and highly compressible graphene aerogels. Adv. Mater. 25, 2219-2223 (2013).

18. Wan, W., Zhao, Z., Hu, H., Gogotsi, Y. \& Qiu, J. Highly controllable and green reduction of graphene oxide to flexible graphene film with high strength. Mater. Res. Bull 48, 4797-4803 (2013).

19. Liu, X. F. \& Antonietti, M. Molten salt activation for synthesis of porous carbon nanostructures and carbon sheets. Carbon 69, 460-466 (2014).

20. Zhang, M., Huang, L., Chen, J., Li, C. \& Shi, G. Ultratough, ultrastrong, and highly conductive graphene films with arbitrary sizes. Adv. Mater. 26, 7588-7592 (2014).

21. Kim, H. W. et al. Selective gas transport through few-layered graphene and graphene oxide membranes. Science 342, 91-95 (2013).

22. Joshi, R. K. et al. Precise and ultrafast molecular sieving through graphene oxide membranes. Science 343, 752-754 (2014).

23. Sun, P. et al. Selective ion penetration of graphene oxide membranes. ACS Nano 7, 428-437 (2012).

24. Zeng, X. et al. Artificial nacre-like papers based on noncovalent functionalized boron nitride nanosheets with excellent mechanical and thermally conductive properties. Nanoscale 7, 6774-6781 (2015).

25. Zeng, X., Ye, L., Sun, R., Xu, J. \& Wong, C. Observation of viscoelasticity in boron nitride nanosheet aerogel. Phys. Chem. Chem. Phys. 17, 16709-16714 (2015) 
26. Zeng, X. et al. Facile preparation of superelastic and ultralow dielectric boron nitride nanosheet aerogels via freeze-casting process. Chem. Mater. 27, 5849-5855 (2015).

27. Yin, J., Li, X., Zhou, J. \& Guo, W. Ultralight three-dimensional boron nitride foam with ultralow permittivity and superelasticity. Nano Lett. 13, 3232-3236 (2013).

28. Rousseas, M. et al. Synthesis of highly crystalline $\mathrm{sp}^{2}$-bonded boron nitride aerogels. ACS Nano 7, 8540-8546 (2013).

29. Wang, Y., Shi, Z. \& Yin, J. Boron nitride nanosheets: large-scale exfoliation in methanesulfonic acid and their composites with polybenzimidazole. J. Mater. Chem. 21, 11371-11377 (2011).

30. Xie, S. -Y. et al. Solubilization of boron nitride nanotubes. Chem. Commun. 3670-3672 (2005)

31. Jung, S. M., Jung, H. Y., Dresselhaus, M. S., Jung, Y. J. \& Kong, J. A facile route for 3D aerogels from nanostructured 1D and 2D materials. Sci. Rep. 2, 849 (2012).

32. Kim, K. S. et al. Hydrogen-catalyzed, pilot-scale production of small-diameter boron nitride nanotubes and their macroscopic assemblies. ACS Nano 8, 6211-6220 (2014).

33. Lei, W., Portehault, D., Dimova, R. \& Antonietti, M. Boron carbon nitride nanostructures from salt melts: tunable water-soluble phosphors. J. Am. Chem. Soc. 133, 7121-7127 (2011).

34. Peng, H. et al. Urea-assisted aqueous exfoliation of graphite for high-quality graphene. Chem. Commun. 51, 4651-4654 (2015).

35. Crimp, M. J. D., Oppermann, A. \& Krehbiel, K. Suspension properties of hexagonal BN powders: effect of $\mathrm{pH}$ and oxygen content. J. Mater. Sci. 34, 2621-2625 (1999).

36. Joni, I. M., Balgis, R., Ogi, T., Iwaki, T. \& Okuyama, K. Surface functionalization for dispersing and stabilizing hexagonal boron nitride nanoparticle by bead milling. Colloids Surf. A Physicochem. Eng. Aspects 388, 49-58 (2011).

37. Sainsbury, T. et al. Self-assembly of gold nanoparticles at the surface of amine- and thiol-functionalized boron nitride nanotubes. J. Phys. Chem. C 111, 12992-12999 (2007).

38. Pentecost, A., Gour, S., Mochalin, V., Knoke, I. \& Gogotsi, Y. Deaggregation of nanodiamond powders using salt- and sugar-assisted milling. ACS Appl. Mater. Interfaces 2, 3289-3294 (2010).

39. Liu, D., Lei, W. \& Chen, Y. Scalable production of winkled and few-layered graphene sheets and their use for oil and organic solvents absorption. Phys. Chem. Chem. Phys. 17, 6913-6918 (2015).

40. Liu, D., Lei, W., Portehault, D., Qin, S. \& Chen, Y. High N-content holey few-layered graphene electrocatalysts: scalable solvent-less production. J. Mater. Chem. A 3, 1682-1687 (2015).

41. Lei, W., Liu, D. \& Chen, Y. Highly crumpled boron nitride nanosheets as sdsorbents: scalable solvent-less production. Adv. Mater. Interfaces 2, 1400529 (2015).

42. Sun, J. F., Wang, M. Z., Zhao, Y. C., Li, X. P. \& Liang, B. Y. Synthesis of titanium nitride powders by reactive ball milling of titanium and urea. J. Alloys Compd. 482, L29-L31 (2009).

43. Li, J. et al. Ultra-light, compressible and fire-resistant graphene aerogel as a highly efficient and recyclable absorbent for organic liquids. J. Mater. Chem. A 2, 2934-2941 (2014).

44. Ji, H. et al. Ultrathin graphite foam: a three-dimensional conductive network for battery electrodes. Nano Lett. 12, 2446-2451 (2012).

45. Chen, Z. et al. Three-dimensional flexible and conductive interconnected graphene networks grown by chemical vapour deposition. Nat. Mater. 10, 424-428 (2011).

46. Worsley, M. A. et al. Mechanically robust 3D graphene macroassembly with high surface area. Chem. Commun. 48, 8428-8430 (2012).
47. Sui, Z., Meng, Q., Zhang, X., Ma, R. \& Cao, B. Green synthesis of carbon nanotube-graphene hybrid aerogels and their use as versatile agents for water purification. J. Mater. Chem. 22, 8767-8771 (2012).

48. Wang, Y., Shi, Z. X. \& Yin, J. Boron nitride nanosheets: large-scale exfoliation in methanesulfonic acid and their composites with polybenzimidazole. J. Mater. Chem. 21, 11371-11377 (2011).

49. Gu, B. \& Wang, H. -T. in Ferroelectrics-Physical Effects. (ed. Lallart, M.) 507-526 (InTech, 2011).

50. Gao, R. et al. High-yield synthesis of boron nitride nanosheets with strong ultraviolet cathodoluminescence emission. J. Phys. Chem. C 113, 15160-15165 (2009).

51. Chen, H. et al. Eu- doped boron nitride nanotubes as a nanometer-sized visiblelight source. Adv. Mater. 19, 1845-1848 (2007).

52. Zhi, C. et al. Large-scale fabrication of boron nitride nanohorn. Appl. Phys. Lett. 87, 063107 (2005).

53. Tang, C., Bando, Y., Zhi, C. \& Golberg, D. Boron-oxygen luminescence centres in boron-nitrogen systems. Chem. Commun. 4599-4601 (2007).

54. Su, C. -Y. et al. Large-scale synthesis of boron nitride nanotubes with ironsupported catalysts. J. Phys. Chem. C 113, 14732-14738 (2009).

55. Silly, M. G. et al. Luminescence properties of hexagonal boron nitride: cathodoluminescence and photoluminescence spectroscopy measurements. Phys. Rev. B 75, 085205 (2007).

56. Zhong, B. et al. Hollow BN microspheres constructed by nanoplates: synthesis, growth mechanism and cathodoluminescence property. Cryst. Eng. Comm. 13, 819-826 (2011).

\section{Acknowledgements}

Financial support from the Australian Research Council under the Discovery programme and Discovery Early Career Researcher Award are acknowledged.

\section{Author contributions}

W.W.L., D.L. and Y.C. conceived the project. W.W.L., D.L., Y.C. and Y.G. designed the experiments. W.W.L., D.L. and S.Q. carried out materials synthesis and performed materials characterization. V.M. carried out $a b$ initio simulations. W.W.L., V.M., Y.G. and Y.C. wrote the manuscript. All authors contributed to analysis of the data and discussions of results.

\section{Additional information}

Supplementary Information accompanies this paper at http://www.nature.com/ naturecommunications

Competing financial interests: The authors declare no competing financial interests.

Reprints and permission information is available online at http://npg.nature.com/ reprintsandpermissions/

How to cite this article: Lei, W. et al. Boron nitride colloidal solutions, ultralight aerogels and freestanding membranes through one-step exfoliation and functionalization. Nat. Commun. 6:8849 doi: 10.1038/ncomms9849 (2015).

This work is licensed under a Creative Commons Attribution 4.0 International License. The images or other third party material in this article are included in the article's Creative Commons license, unless indicated otherwise in the credit line; if the material is not included under the Creative Commons license, users will need to obtain permission from the license holder to reproduce the material To view a copy of this license, visit http://creativecommons.org/licenses/by/4.0/ 